\title{
ПРОГРАММА МОДЕАИРОВАНИЯ ТЕМПЕРАТУРЫ ПРОВОДА И ПОТЕРЬ МОЩНОСТИ НА ОСНОВЕ УЧЕТА РЕЖИМНЫХ И АТМОСФЕРНЫХ ФАКТОРОВ
}

\author{
А.Б. Баламетов 1, д.m.н., npoфpeccop, balametov.azniie@gmail.com \\ Э.Д. Халилов ${ }^{1}$, к.т.н., зав. отделом \\ М.П. Байрамов 2, к.т.н., ст. преподаватель \\ К.А. Агаханова ${ }^{3}$, докторант, ст. лаборант
}

${ }_{1}$ Азербайджанский научно-исследовательский и проектно-изыскательский институт энергетики, ул. Зардаби Гасанбека, 94, г. Баку, Аз1012, Азербайджан

2 Азербайджанский государственный университет нефти и промышленности, просп. Азадльг, 20, г. Баку, АZ1010, Азербайджан

${ }_{3}^{3}$ Сумгаитский государственный университет, 43-й квартал, AZ5008, г. Сумгаит, Азербайджан

В современных условиях в связи с ростом электропотребления и заметными климатическими изменениями повысился интерес к расчетам допустимых механических и токовых нагрузок на провода воздушных линий. Максимальное использование их пропускной способности может быть достигнуто при наличии достоверной информации о состоянии линии. Повысить точность расчета потерь электроэнергии в воздушных линиях электропередачи можно, определив активное сопротивление проводов с учетом протекающего по линиям рабочего тока, температуры окружающего воздуха, скорости ветра и теплоты солнечного излучения.

В этих условиях очень важно иметь ПО для оценки и непрерывного контроля температуры провода при различных погодных условиях, достоверно определять допустимую токовую нагрузку и иметь возможность при возникновении недопустимой перегрузки линии, в том числе в ремонтных режимах, выполнять мероприятия по разгрузке остающихся в работе воздушных линий.

Современные программы расчета режимов энергосистем не включают в себя уравнения тепловых балансов проводов воздушных линий. Это приводит к невозможности учета их температурного режима из-за несоответствия активных сопротивлений проводов их температуре. Авторами данной статьи разработаны алгоритм и программа расчета удельного активного сопротивления проводов воздушных линий с учетом температуры воздуха, рабочего тока, скорости ветра и солнечной радиации. Проведена количественная оценка влияния тока нагрузки, температуры окружающей среды, солнечной радиации и скорости ветра на активное сопротивление проводов воздушных линий. Приведены результаты расчета предельных токовых нагрузок для проводов с активным сопротивлением.

На основе проведенных расчетов по оценке влияния тока нагрузки, температуры окружающей среды, солнечной радиации и скорости ветра на активное сопротивление проводов воздушных линий установлено, что при расчете потерь электроэнергии для проводов без учета температурной зависимости сопротивления относительные погрешности могут достигать $26 \%$ и более, что недопустимо. Поэтому требуется автоматическая регистрация температуры провода для повышения точности расчета потерь активной мощности и энергии.

Ключевые слова: воздушная линия, уравнение теплового баланса, рабочий ток, погодные условия, температура провода, численное моделирование, мониторинг.

В связи с увеличением энергопотребления нагрузки на воздушные линии (ВЛ) электропередачи растут довольно высокими темпами, а сетевое строительство пока явно отстает. Максимальное использование пропускной способности ВЛ может быть достигнуто при наличии достоверной информации об их состоянии, а также актуальных данных о температуре проводов и плотности протекающего по ним тока.

Одним из направлений повышения точности расчета переменных потерь электроэнергии в ВЛ является определение активных сопротивлений проводов с учетом протекающего по линиям рабочего тока, температуры окружающего воздуха, скорости ветра и теплоты солнечного излучения.

В соответствии с [1-6], при определении технологических потерь электроэнергии по электросетям необходимо учитывать температуру провода, влияющую на величину активного сопротивления.
Температура провода, в свою очередь, зависит от режимных и климатических факторов: плотности тока, температуры окружающего воздуха $t_{\mathrm{B}}$, силы и направления ветра, интенсивности солнечной радиации и т.д. [1-14].

Удельное активное сопротивление проводов ВЛ

$$
R=R_{20} \cdot\left[1+\alpha \cdot\left(t_{\text {пр. }}-20\right)\right\rfloor,
$$

где $R_{20}$ - удельное активное сопротивление при температуре провода $20^{\circ} \mathrm{C}$, Ом/км; $\alpha=0.004$ - температурный коэффициент электрического сопротивления сталеалюминиевых проводов, 1/град; $t_{\text {nр. }}-$ температура провода, ${ }^{\circ} \mathrm{C}$.

Температура провода в формуле (1), как правило, неизвестна. Она зависит от большого числа факторов и прежде всего от протекающего по проводу электрического тока, температуры окружающего воздуха и скорости ветра. В традиционных 
расчетах переменных потерь электроэнергии активные сопротивления принимаются по справочникам, в которых они приведены к температуре $20{ }^{\circ} \mathrm{C}$. В лучшем случае активные сопротивления пересчитываются с учетом температуры воздуха $t_{\mathrm{B}}$.

Цель авторов данной статьи - разработка алгоритма и оценка влияния тока нагрузки, температуры провода, солнечной радиации на активное сопротивление проводов при скорости ветра $0.5 \div 10 \mathrm{M} / \mathrm{c}$, а также определение диапазонов погрешностей расчета годовых переменных потерь электроэнергии.

Зависимость температуры провода от параметров внешней среды может быть представлена в виде уравнения теплового баланса провода, коэффициенты которого для различных случаев впервые были получены Бургсдорфом [1].

Уравнение теплового баланса для установившегося теплового режима записывается следующим образом:

$$
\begin{aligned}
& I^{2} \cdot R_{20} \cdot\left[1+\alpha \cdot\left(t_{\text {пр. }}-20\right)\right]+W_{\text {с }}= \\
& =\pi d_{\text {пр. }}\left(\alpha_{\text {к }}+\alpha_{\text {л }}\right)\left(t_{\text {пр. }}-t_{\text {в }}\right),
\end{aligned}
$$

где $I$ - ток линии, А; $R_{20}$ - удельное активное сопротивление при температуре провода $20{ }^{\circ} \mathrm{C}$, Ом/м; $t_{\mathrm{B}}$ - температура воздуха, ${ }^{\circ} \mathrm{C} ; \alpha_{\mathrm{\kappa}}, \alpha_{\text {л }}-$ коэффициенты теплоотдачи провода при конвективном и лучистом теплообмене, $\mathrm{BT} /\left(\mathrm{M}^{2} \cdot{ }^{\circ} \mathrm{C}\right) ; W_{\mathrm{c}}$ - теплота солнечного излучения, поглощаемая 1 м провода в единицу времени, Вт; $d_{\text {пр }}$ - диаметр провода, м.

В уравнении (2) левая часть представляет собой поглощаемую теплоту солнечного излучения и теплоту от токовой нагрузки, а правая часть состоит из суммы конвективных потерь и потерь на радиацию.

Коэффициент теплоотдачи лучеиспусканием определяется по формуле Стефана-Больцмана $[5,6,9]$ :

$$
Q_{\text {л }}=\varepsilon C_{0}\left(273+t_{\text {пр. }}\right)^{4} S,
$$

где $\varepsilon-$ степень черноты поверхности провода для окисленного алюминия, равная 0.13 отн. ед.; $C_{0}-$ коэффициент излучения абсолютно черного тела, равный $5.67 \cdot 10^{-8}$ Вт/м ${ }^{2} ; S-$ площадь поверхности провода, м $^{2}$.

Потери мощности при теплоотдаче конвекцией [2] определяем по формуле

$$
Q_{\mathrm{K}}=\alpha_{k}\left\lfloor\left(t_{\text {пр. }}-t_{\text {рад. }}\right)-t_{\text {в }}\right\rfloor S
$$

где $\alpha_{\kappa}-$ коэффициент теплоотдачи конвекцией, $\mathrm{BT} /\left(\mathrm{M}^{2}{ }^{\circ} \mathrm{C}\right) ; t_{\text {рад. }}-$ температура нагрева солнечной радиацией, ${ }^{\circ} \mathrm{C}$.

Коэффициент теплоотдачи конвекцией определяем по формуле

$$
a_{k}=0.13057 \cdot\left(\frac{k_{v} v d_{\text {пр. }}}{a}\right)^{0.71719} \frac{\lambda_{a}}{d_{\text {пр. }}},
$$

где $k_{v}$ - коэффициент, учитывающий влияние угла направления ветра к оси ВЛ, равный $0.5 ; v$ - ско- рость ветра, м/с; $a$ - коэффициент температуропроводности воздуха, равный $18.8 \cdot 10^{-6} \mathrm{M}^{2} / \mathrm{c} ; \lambda_{\text {в }}$ - теплопроводность воздуха, равная $0.0244 \mathrm{BT} /\left(\mathrm{M}^{\circ} \mathrm{C}\right)$.

Из уравнений (2-5) для тока $[5,6]$ можно получить

$$
I=\sqrt{\frac{\varepsilon C_{0}\left(273+t_{\text {пр. }}\right)^{4} \pi d+\alpha_{k}\left[\left(t_{\text {пр. }}-t_{\text {рад. }}\right)-t_{\text {в }}\right] \pi d}{0.95 \cdot R_{20} \cdot\left[1+\alpha \cdot\left(t_{\text {пр. }}-20\right)\right]}} \text {. (6) }
$$

Получение зависимости температуры провода от тока нагрузки, температуры воздуха и скорости ветра в явном виде из уравнения (6) не удается. В связи с этим для решения уравнения (6) относительно температуры провода и получения аналитических зависимостей от тока нагрузки, температуры воздуха и скорости ветра применяется следующий алгоритм.

1. Получение зависимости тока от температуры провода, скорости ветра и температуры воздуха для заданного провода по формуле (6) для конкретных значений $t_{\mathrm{B}}, t_{\text {рад., }} v$ в табличном виде.

2. Аппроксимация полученных зависимостей в областях температур от $-40{ }^{\circ} \mathrm{C}$ до $+40{ }^{\circ} \mathrm{C}$ полиномом третьей степени:

$$
t_{\text {пр. }}=a_{0}+a_{1} \cdot I+a_{2} \cdot I^{2}+a_{3} \cdot I^{3} .
$$

В связи с зависимостью коэффициента теплоотдачи от температуры провода температуру неизолированных проводов можно вычислить по формуле (6) и методом последовательных приближений на основе уравнения

$$
t_{\text {nр. }}^{[k+1]}=\frac{\pi d_{\text {пр. }}\left\lfloor\alpha_{\text {к }}\left(t_{\text {nр. }}^{[k]}\right)\right\rfloor-\alpha_{\text {л }}\left(t_{\text {np. }}^{[k]}\right)-I^{2} \cdot R_{20} \cdot(20 \alpha-1)+W_{c}}{\pi d_{\text {пр. }} \cdot \alpha_{\kappa}\left(\begin{array}{c}
t_{\text {nр. }}^{[k]} \\
)
\end{array}\right)-I^{2} \cdot \alpha} .
$$

Здесь к - номер итерации. По формуле (8) можно вычислить температуру провода при известном токе и различных погодных условиях.

Для определения теплоты солнечного излучения, поглощаемого проводом, существует несколько моделей [3-7]. Наиболее полно эти вопросы рассмотрены в [5-8]. Теплота солнечного излучения определяется уравнением

$$
W_{c}=3 \cdot \varepsilon_{\Pi} \cdot K_{\mathrm{H}} \cdot d_{\text {пр. }} \cdot W_{c} \cdot \sin \psi_{c},
$$

где $\varepsilon_{\text {п }}=0,6$ - коэффициент поглощения провода; $k_{\mathrm{H}}-$ коэффициент, учитывающий влияние высоты над уровнем моря; $W_{c}-$ интенсивность суммарной радиации (прямой и отраженной), Вт/м²; $\psi_{c}-$ активный угол наклона солнечных лучей.

Из уравнений теплового баланса (6) провода следует, что минимальное допустимое значение

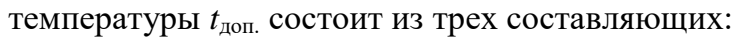

$$
t_{\text {доп. }}=t_{I}+t_{\mathrm{B}}+t_{\text {рад., }}
$$

где $t_{I}$ - составляющая от нагрева провода током нагрузки за счет потерь энергии в активном сопротивлении провода.

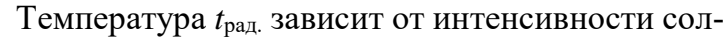
нечной радиации, высоты и плотности облаков. Интенсивность радиации солнца изменяется в течение года. По его состоянию в сентябре от 14 ч 
30 мин. до 15 ч 30 мин. при синем небе и отсутствии ветра для проводов АС в [3] получено уравнение

$$
t_{\text {рад. }}=K_{\tau} \cdot K_{\tau}^{\prime} \cdot K_{\text {рад. }} \cdot d^{0.44152} .
$$

Здесь $K_{\text {рад. }}=92.0375{ }^{\circ} \mathrm{C} / \mathrm{M}$. В период с 20 до 24 часов и с 24 до 7 часов принимается $t_{\text {рад. }}=0$.

Для проводов разных марок для учета солнечной радиации в июне для времени с 11 до 13 ч (наибольший нагрев) при расчете в формулу (11) вводятся коэффициенты $K_{\tau}=1.15, K_{\tau}{ }^{\prime}=1$ при синем небе и $K_{\tau}{ }^{\prime}=0,5$ при сером небе и видимом солнце.

Определение допустимой температуры провода. В настоящее время длительно допустимая температура провода для нормального режима принята равной $70{ }^{\circ} \mathrm{C}$.

Для утяжеленного режима температура провода выше $70{ }^{\circ} \mathrm{C}$, но ниже максимально допустимой по условиям механической прочности $90{ }^{\circ} \mathrm{C}$ и допустимой по условию сохранения габаритов. Аварийный режим по токовой перегрузке - установившаяся температура провода превышает $100^{\circ} \mathrm{C}$ или допустимую по условию сохранения габаритов или гололедная нагрузка превышает допустимую.

Максимально допустимый ток провода [6] определяется из выражения

$$
I=\sqrt{\frac{\left(\alpha_{\text {л }}+\alpha_{k}\right) \cdot \pi d \cdot\left(t_{\text {пр. }}-t_{\text {рад. }}\right)-W_{c}}{R_{20} \cdot\left[1+\alpha \cdot\left(t_{\text {пр. }}-20\right)\right]}} .
$$

С ростом скорости ветра $V$ (и $\left.a_{\kappa}\right)$ значение $I_{\text {доп. }}$ увеличивается, однако для повышения надежности допустимый ток вычисляют для наименьшей нормативной скорости ветра $(0.5 \mathrm{M} / \mathrm{c})$ и его продольном направлении $\left(k_{V}=0,5\right)$.

Для моделирования изменения температуры провода во времени уравнение теплового баланса записывается в дифференциальной форме и расчет ведется методами численного интегрирования.

Время нарастания температуры провода. Температурная характеристика переходного процесса может быть рассчитана в соответствии с методом, описанным в $[8,9]$.

Для нагрева провода от температуры $t_{1}$ с током $I_{1}$ до $t_{2}$ с током $I_{1}$ требуется время, определяемое минутами. В программе реализован следующий алгоритм нарастания температуры провода в зависимости от времени.

Постоянная времени рассчитывается по формуле $\tau=\frac{\left(t_{2}-t_{1}\right) \cdot m C_{\text {пр. }}}{R_{t} \cdot\left(t_{2}-t_{1}\right) \cdot\left(I_{2}^{2}-I_{1}^{2}\right)}$.

Характер нарастания температуры провода до установившейся температуры определяется переходным процессом по формуле

$$
t_{\text {пр. }}(\mathrm{l})=t_{1}+\left(t_{2}-t_{1}\right) \cdot\left(1-e^{-\left(\frac{t}{\tau}\right)}\right) .
$$

В Азербайджанском научно-исследовательском и проектно-изыскательском институте энергетики

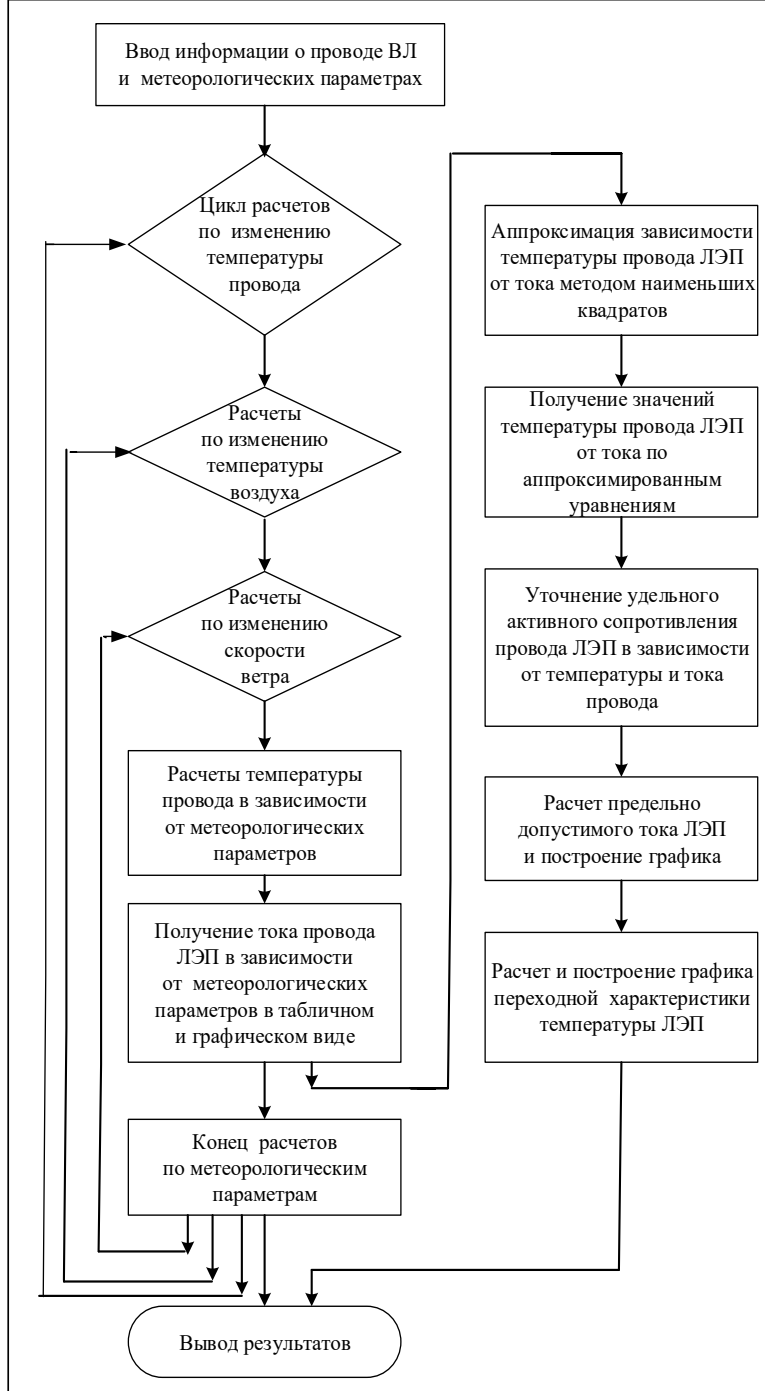

Рис. 1. Программа моделирования температуры и активного сопротивления провода ВЛ в зависимости от тока нагрузки провода и атмосферных факторов

Fig. 1. The program for modeling temperature and active resistance of an aerial wire depending on the load current and atmospheric factors

разработан алгоритм, реализованный в виде программы моделирования температуры провода с учетом тока нагрузки ВЛ и влияния атмосферных факторов. Уравнения (1-14) составляют основу комплекса алгоритмов и программ данного института. Блок-схема программы и экранные формы приведены на рисунках 1 и 2.

Программа использовалась при моделировании технических потерь электроэнергии на Азербайджанской ЭЭС и мониторинга ВЛ 110 кВ для определения допустимой токовой нагрузки по условию передачи мощности электрической станции Шимал-1 по пяти линиям 110 кВ и введения ограничений по мощности и нашла практическое применение. 


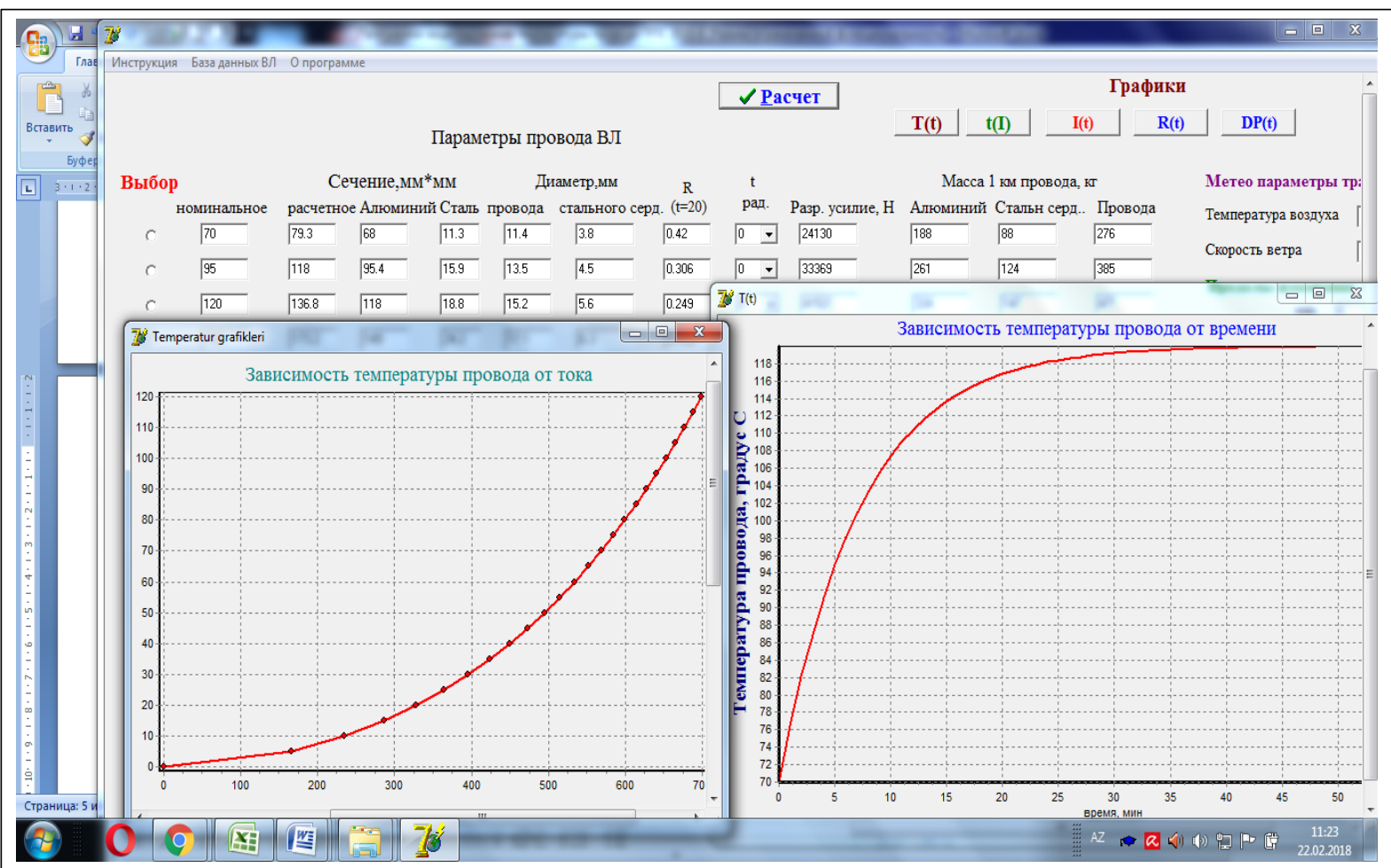

Рис. 2. Экранные формы программы моделирования температуры провода

Fig. 2. Screen forms of the wire temperature simulation program

С использованием программы проведены расчеты для провода АС 240/39 с удельным активным сопротивлением $R_{20}=0.121$ Ом/км и диаметром провода $d=21.6$ мм. Получены зависимости тока от температуры провода, скорости ветра и температуры воздуха по формуле (7) при $v=0,5 \mathrm{м} / \mathrm{c}$ и $t_{\text {рад. }}=0{ }^{\circ} \mathrm{C}$ в области температур от -40 до $+40{ }^{\circ} \mathrm{C}$. После подстановки численных значений $R_{20}, d$ и $v$ в формулу (7) получаем значения токовых нагрузок провода при заданной температуре воздуха и температуре провода, которые представлены в таблице 1.

Аппроксимация кубической зависимости тока от температуры провода, скорости ветра и температуры воздуха имеет достаточную для практики точность. В таблице 2 представлены коэффициенты кубической зависимости из формулы (7) для расчета температуры провода АС 150/24 при $v=0.5 \mathrm{M} / \mathrm{c}$ и $t_{\text {рад. }}=0{ }^{\circ} \mathrm{C}$, полученные по разработанной программе.

Токовые нагрузки провода

Таблица 1

Wire current load

Table 1

\begin{tabular}{|l|c|c|c|c|c|c|c|c|c|}
\hline \multirow{2}{*}{$\begin{array}{c}\text { Температура } \\
\text { провода, }{ }^{\circ} \mathbf{C}\end{array}$} & \multicolumn{7}{|c|}{ Токовые нагрузки А при температура воздуха, ${ }^{\circ} \mathbf{C}$} \\
\hline & $-\mathbf{4 0}$ & $\mathbf{- 3 0}$ & $\mathbf{- 2 0}$ & $\mathbf{- 1 0}$ & $\mathbf{0}$ & $\mathbf{1 0}$ & $\mathbf{2 0}$ & $\mathbf{3 0}$ & $\mathbf{4 0}$ \\
\hline-40 & 129.87 & & & & & & & & \\
\hline-30 & 286.34 & 137.68 & & & & & & & \\
\hline-20 & 375.87 & 285.04 & 145.65 & & & & & & \\
\hline-10 & 442.22 & 371.83 & 284.52 & 153.77 & & & & & \\
\hline 0 & 495.49 & 436.69 & 368.63 & 284.73 & 162.04 & & & & \\
\hline 10 & 540.10 & 489.06 & 432.03 & 366.22 & 285.64 & 170.47 & & & \\
\hline 20 & 578.47 & 533.10 & 483.49 & 428.18 & 364.57 & 287.20 & 179.03 & & \\
\hline 30 & 612.10 & 571.12 & 526.97 & 478.75 & 425.11 & 363.63 & 289.37 & 187.75 & \\
\hline 40 & 642.02 & 604.57 & 564.63 & 521.65 & 474.79 & 422.76 & 363.37 & 292.14 & 196.60 \\
\hline 50 & 668.95 & 634.41 & 597.87 & 558.94 & 517.10 & 471.56 & 421.12 & 363.75 & 295.45 \\
\hline 60 & 693.42 & 661.33 & 627.60 & 591.96 & 554.02 & 513.29 & 469.03 & 420.14 & 364.76 \\
\hline 70 & 715.84 & 685.86 & 654.51 & 621.57 & 586.80 & 549.82 & 510.18 & 467.18 & 419.80 \\
\hline 80 & 736.53 & 708.39 & 679.07 & 648.44 & 616.28 & 582.35 & 546.32 & 507.74 & 465.97 \\
\hline 90 & 755.75 & 729.22 & 701.69 & 673.03 & 643.09 & 611.70 & 578.60 & 543.49 & 505.95 \\
\hline
\end{tabular}


Dependence of conductor temperature on current and air temperature

\begin{tabular}{|c|c|c|c|c|c|}
\hline \multirow[t]{2}{*}{ № } & \multirow{2}{*}{$\begin{array}{c}\text { Температура } \\
\text { воздуха, }{ }^{\circ} \mathrm{C}\end{array}$} & \multicolumn{4}{|c|}{ Коэффициенты кубической зависимости (7) } \\
\hline & & a0 & a1 & a2 & $\mathrm{a}^{2} * 10^{-4}$ \\
\hline 1 & -40 & 500.387432 & 5.519205 & -0.064520 & 3.99 \\
\hline 2 & -30 & 435.373966 & 6.677718 & -0.076860 & 4.44 \\
\hline 3 & -20 & 360.459091 & 8.098142 & -0.091778 & $4 . .97$ \\
\hline 4 & -10 & 272.718601 & 9.859973 & -0.110021 & 5.61 \\
\hline 5 & 0 & 168.088545 & 12.073962 & -0.132636 & 6.40 \\
\hline 6 & 10 & 40.776746 & 14.896292 & -0.161074 & 7.37 \\
\hline 7 & 20 & -117.750714 & 18.559679 & -0.197511 & 8.61 \\
\hline 8 & 30 & -320.239048 & 23.407944 & -0.245115 & 10.20 \\
\hline 9 & 40 & -587.142778 & 30.001478 & -0.309140 & 12.31 \\
\hline
\end{tabular}

Представление графика кусочно-нелинейной аппроксимации делением на две части $t_{\mathrm{B}} \leq 0$ и $t_{\mathrm{B}}>0$ и применение для аппроксимации полинома второй степени имеют определенные удобства и практически приемлемую точность по сравнению с результатами полиномов более чем во второй степени.

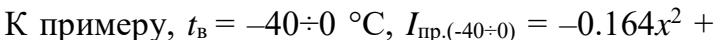
$+2.3118 x+490.58$ и $t_{\mathrm{B}}=0 \div 90{ }^{\circ} \mathrm{C}, I_{\text {пр. }(0 \div 90)}=$ $=-0.0149 x^{2}+4.1767 x+498.45$.

Сравнение с полиномом 3-й степени показывает, что СКО имеет значение $4.35 \%$, тогда как кусочно-нелинейная аппроксимация полинома второй степени делением на две части имеет лучшую точность: первая часть имеет СКО 3 \%, а вторая $0.21 \%$.

По формуле (7) для коэффициентов таблицы 2 рассчитана температура провода в зависимости от тока в диапазоне 110-350 А для температуры воздуха от $-40{ }^{\circ} \mathrm{C}$ до $+40{ }^{\circ} \mathrm{C}$.

График зависимости допустимого тока провода АC $240 / 39$ при температуре воздуха $-40{ }^{\circ} \mathrm{C}, 0^{\circ} \mathrm{C}$ и $40{ }^{\circ} \mathrm{C}$ и скорости ветра $v=0.5 \mathrm{~m} / \mathrm{c}$ приведен на рисунке 3.

Для получения зависимостей активного сопротивления от тока нагрузки, температуры воздуха и скорости ветра подставим температуру провода из таблицы 2 в формулу (1).

На рисунке 4 представлен график зависимости удельного активного сопротивления провода АC $240 / 39$ от тока при температуре воздуха $-40{ }^{\circ} \mathrm{C}$, скорости ветра $0.5 \mathrm{M} /$ сек. и $t_{\text {рад. }}=0{ }^{\circ} \mathrm{C}$.

Зависимость температуры провода от скорости ветра и тока провода АС150 при $t_{\mathrm{B}}=20^{\circ} \mathrm{C}$ и $t_{\text {рад. }}=$ $=0{ }^{\circ} \mathrm{C}$ показана на рисунке 5 .

Погрешности расчета сопротивлений линий электропередачи могут быть достаточно большими и составлять от -8 до $24 \%$ и более.

В таблице 3 представлены результаты расчетов суточных переменных потерь электроэнергии по

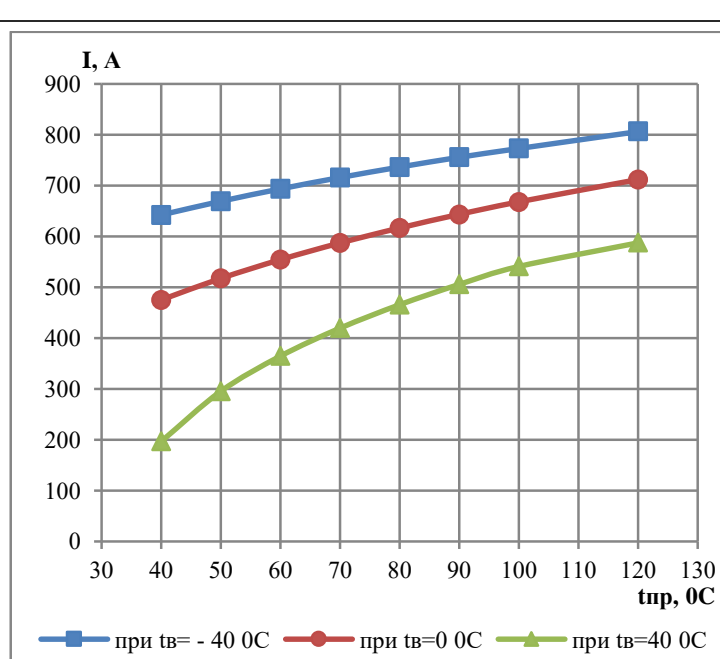

Рис. 3. Графики допустимого тока провода АС 240/39

Fig. 3. Graphs of permissible wire current AC 240/39

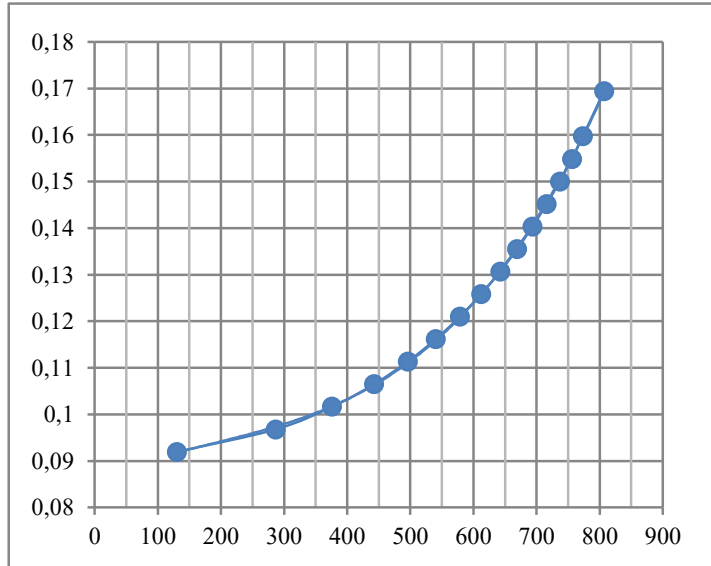

Рис. 4. Зависимость удельного активного сопротивления провода АС 240/39 от тока

Fig. 4. Dependence of the specific active resistance of the wire AC $240 / 39$ on current 


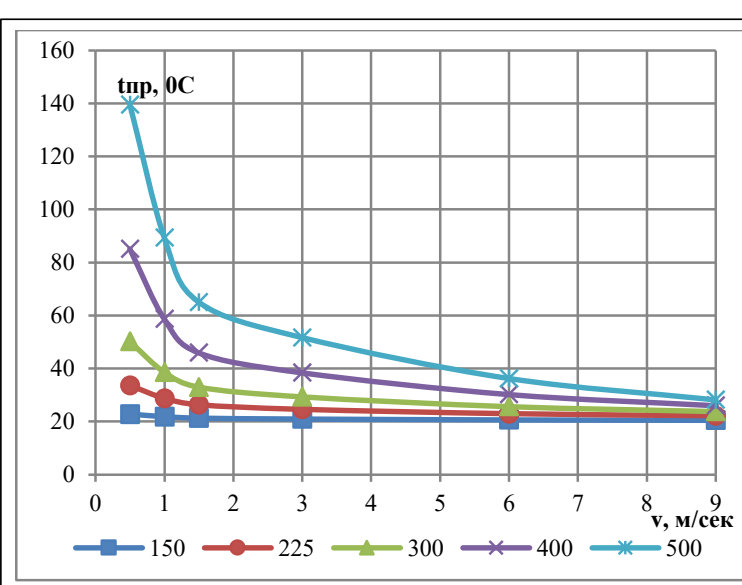

Рис. 5. Зависимость температуры провода от скорости ветра и тока провода

Fig. 5. Dependence of wire temperature on wind speed and wire current

формуле (10) с учетом диапазона токов для проводов АС 150/24.

Таблица 3

\section{Переменные потери электроэнергии} провода АC-150/24

Variable power losses of AC-150/24 wire

Table 3

\begin{tabular}{|c|c|c|c|c|c|}
\hline \multirow{2}{*}{\begin{tabular}{|c} 
Сред- \\
ний ра- \\
бочий \\
ток, А
\end{tabular}} & \multicolumn{4}{|c|}{$\begin{array}{c}\text { Потери электроэнергии за летние сутки, } \\
\text { кВт/час }\end{array}$} & \multirow{2}{*}{$\begin{array}{c}\text { По- } \\
\text { греш- } \\
\text { ность, } \\
\%\end{array}$} \\
\hline & $\begin{array}{c}\text { при } \\
t_{\mathrm{B}}=\mathbf{2 0}{ }^{\circ} \mathrm{C}\end{array}$ & $\begin{array}{c}\text { ночь } \\
t_{\mathrm{B}}=30{ }^{\circ} \mathrm{C}, \\
t_{\mathrm{pad} .}=\mathbf{0}\end{array}$ & \begin{tabular}{c|} 
день \\
$t_{\mathrm{B}}=40^{\circ} \mathrm{C}$, \\
$t_{\mathrm{par}}=17.6^{\circ} \mathrm{C}$
\end{tabular} & сутки & \\
\hline 150 & 320.76 & 166.66 & 184.08 & 350.74 & -9.35 \\
\hline 200 & 570.24 & 300.39 & 335.69 & 636.09 & -11.55 \\
\hline 250 & \begin{tabular}{|l|}
891.00 \\
\end{tabular} & 476.06 & \begin{tabular}{|l|}
541.99 \\
\end{tabular} & \begin{tabular}{|l|}
1018.05 \\
\end{tabular} & \begin{tabular}{|l|}
-14.26 \\
\end{tabular} \\
\hline 300 & 1283.04 & 695.79 & 813.22 & 1509.01 & -17.61 \\
\hline 350 & 1746.36 & 962.22 & \begin{tabular}{|l|}
1164.00 \\
\end{tabular} & 2126.22 & -21.75 \\
\hline \begin{tabular}{|l|}
400 \\
\end{tabular} & 2280.00 & 1279.00 & 1613.00 & 2892.00 & -26.84 \\
\hline
\end{tabular}

Рассчитана переходная температура провода AC 240/39 по параметрам погоды: $v=0.5$ м/сек., $t_{\mathrm{B}}=40{ }^{\circ} \mathrm{C}, W_{c}=900 \mathrm{BT} / \mathrm{M}$. При этом происходит изменение тока от начального $I_{\text {нач. }}=70$ А до конечного $I_{\text {кон. }}=100 \mathrm{~A}$.

Постоянная времени рассчитана по формуле (13): $\tau=\frac{(120-70) \cdot 704.604}{17.11 \cdot 10^{-4} \cdot(120-70) \cdot\left(789^{2}-387^{2}\right)}=435.626$ сек.

\section{Результаты}

В современных условиях эксплуатации электрических сетей важное значение имеют ПО для мониторинга температуры провода с учетом погодных условий, возможность повышения пропускной способности линии и выполнение мероприятий по разгрузке для недопущения перегрузки линий.

Авторами разработаны алгоритм и программа расчета удельного активного сопротивления приводов ВЈI с учетом температуры воздуха, рабочего тока, скорости ветра и солнечной радиации. Проведена количественная оценка влияния тока нагрузки, температуры окружающей среды, солнечной радиации и скорости ветра на активное сопротивление проводов ВЛ.

Алгоритм и программа протестированы на примере разных марок проводов АС 70/11, АС 95/16, AC 120/19 и АC 150/24, АC 240/39. Приведены результаты расчета зависимости удельного активного сопротивления от температуры воздуха, рабочего тока, солнечной радиации, предельных токовых нагрузок, характера нарастания температуры провода до установившейся температуры, определяющейся переходным процессом.

Установлено, что при расчете потерь электроэнергии для проводов без учета температурной зависимости сопротивления относительные погрешности могут достигать $26 \%$ и более, что недопустимо. Поэтому в условиях эксплуатации необходима автоматическая регистрация температуры провода для повышения точности расчета потерь активной мощности и энергии.

\section{Лumepamypa}

1. Бургсдорф В.В., Никитина Л.Г. Определение допустимых токов нагрузки воздушных линий электропередачи по току их проводов // Электричество. 1989. № 11. С. 1-8.

2. Зарудский Г.К., Зиннер Л.Э., Сыромятников С.Ю. Расчет температуры проводов воздушных линий электропередачи СВН на основе метода критериального планирования эксперимента // Вестн. МЭИ. 1997. № 12. С. 85-90.

3. Никифоров Е.П. Предельно допустимые токовые нагрузки на провода действующих ВЛ с учетом нагрева проводов солнечной радиацией // Электрические станции. 2006. № 7. C. $56-59$.

4. Воротницкий В.Э., Туркина О.В. Оценка погрешностей расчета переменных потерь электроэнергии в ВЛ из-за неучета метеоусловий // Электрические станции. 2008. № 10. С. 42-49.

5. Левченко И.И., Сацук Е.И. Нагрузочная способность воздушных линий электропередачи в экстремальных погодных условиях // Электричество. 2008. № 4. С. 2-8.

6. Фигурнов Е.П., Жарков Ю.И., Петрова Т.Е. Уточненная методика расчета нагрева проводов воздушных линий электропередачи // Электрические станции. 2013. № 9. С. 54-59.

7. Guide for Thermal Rating Calculations of Overhead Lines. WG B2.43, Tech. Rep. 601, CIGRE, 2014, 95 p.

8. Механошин Б.И., Шкапцов В.А., Васильев Ю.А. Повышение эффективности использования существующих ВЛ на основе анализа их технического состояния и данных мониторинга температуры проводов // Электро. 2007. № 6. С. 37-41.

9. Герасименко А.А., Тимофеев Г.С., Тихонович А.В. Учет схемно-режимных и атмосферных факторов при расчете технологических потерь электроэнергии в распределительных сетях // Журн. Сибирского федер. ун-та. Сер.: Техника и технологии. 2008. Т. 1. № 2. С. 188-206.

10. Петрова Е.В., Бубенчиков А.А., Кириченко Н.В., Птицына Е.В. Разработка комплекса программ сравнения методов расчета потерь электрической энергии в воздушных линиях электроэнергетических систем с учетом режимных и климатических факторов // Омский науч. вестн. 2011. № 3. С. 221-225.

11. Баламетов А.Б., Байрамов М.П. Моделирование температуры провода для расчета потерь электроэнергии воздушных линий // Проблемы энергетики. 2013. № 2. С. 4-12.

12. Cecchi V., Leger A.St., Karen M., Nwankpa C.O. Incorporating temperature variations into transmission-line models. IEEE Transactions on Power Delivery, 2011, vol. 26, i. 4, pp. 2189-2196.

13. Mo Y., Zhou X., Wang Ya., Liang L. Study on operating status of overhead transmission lines based on wind speed variation. Progress In Electromagnetics Research M, 2017, vol. 60, pp. 111-120. 
14. Rahman M., Kiesau M., and Cecchi V. Investigating the impacts of conductor temperature on power handling capabilities of transmission lines using a multi-segment line model. Proc. SoutheastCon, 2017, pp. 1-7.
Software \& Systems

DOI: $10.15827 / 0236-235 X .122 .396-402$
Received 28.02.18

2018, vol. 31, no. 2, pp. 396-402

\title{
A PROGRAM OF MODELING WIRE TEMPERATURE AND POWER LOSSES BASED ON OPERATION AND ATMOSPHERIC FACTORS
}

\author{
A.B. Balametov ${ }^{1}$, Dr.Sc. (Engineering), Professor, balametov.azniie@gmail.com \\ E.D. Khalilov ${ }^{1}$, Ph.D. (Engineering), Head of Department \\ M.P. Bayramov ${ }^{2}$, Ph.D. (Engineering), Senior Lecturer \\ K.A. Agakhanova ${ }^{3}$, Doctoral Student, Senior Laboratory Assistant \\ ${ }^{1}$ Azerbaijan Scientific-Research and Design-Prospecting Power Engineering Institute, Zardabi St. 94, Baku, Aз1012, Azerbaijan \\ ${ }^{2}$ Azerbaijan State University of Oil and Industry, Azadlyg Ave. 20, Baku, AZ1010, Azerbaijan \\ ${ }^{3}$ Sumgait State University, 43rd block, AZ5008, Sumgait, Azerbaijan
}

Abstract. In modern conditions, due to the growth of power consumption and noticeable climatic changes, the interest in calculating admissible mechanical and current loads on the aerial wires has been increased. The maximum use of overhead line capacity can be achieved with reliable information on the line state. The accuracy of calculating electric power losses in overhead power transmission lines might be increased by determining active resistance of wires taking into account the operating current flowing along the lines, the ambient air temperature, wind speed and solar radiation heat.

In these conditions, it is very important to have software for evaluating and continuously monitoring wire temperature under various weather conditions, to reliably determine the permissible current load and to be able, if unacceptable line overload occurs, including repair modes, to carry out measures for unloading the remaining work of an aerial line.

Modern programs for calculating power systems modes do not include thermal balance equations of aerial lines. This leads to the impossibility of taking into account their temperature conditions due to the mismatch of wire active resistance to their temperature. The authors have developed an algorithm and a program for calculating the specific active resistance of aerial lines wires taking into account the air temperature, working current, wind speed and solar radiation. They also carried out a quantitative assessment of the load current influence, ambient temperature, solar radiation and wind speed on the active resistance of aerial wires. There are the results of calculation of limited current loads for aerial wires.

Based on the calculations to evaluate the effect of load current, ambient temperature, solar radiation and wind speed on active resistance of aerial wires, it is established that when calculating power losses for wires without considering the resistance temperature dependence, relative errors can reach $26 \%$ or more, which is not permissible. Therefore, it is required to automatically detect the wire temperature in order to improve the accuracy of the calculation of active power and energy losses.

Keywords: aerial line, heat balance equation, operating current, weather conditions, wire temperature, numerical simulation, monitoring.

\section{References}

1. Burgsdorf V.V., Nikitina L.G. Determination of permissible current of aerial power lines load by current of their wires. Elektrichestvo [Electricity]. 1989, no. 11, pp. 1-8 (in Russ.).

2. Zarudsky G.K., Zinner L.E., Syromyatnikov S.Yu. Calculation of the temperature of SVN overhead power transmission wires based on the method of criterial experiment planning. Vestn. MEI [MPEI Vestn.]. 1997, no. 12, pp. 85-90 (in Russ.).

3. Nikiforov E.P. Maximum permissible current loads of active aerial wires, taking into account heating of wires by solar radiation. Elektricheskie stantsii [Electric Power Plants]. 2006, no. 7, pp. 56-59 (in Russ.).

4. Vorotnitsky V.E., Turkina O.V. Estimation of errors in calculation of variable electric energy losses in aerial lines due to non-meteorological conditions. Elektricheskie stantsii [Electric Power Plants]. 2008, no. 10, pp. $42-49$ (in Russ.).

5. Levchenko I.I., Satsuk E.I. Load capacity of aerial transmission lines in extreme weather conditions. Elektrichestvo [Electricity]. 2008, no. 4, pp. 2-8 (in Russ.).

6. Figurnov E.P., Zharkov Yu.I., Petrova T.E. A refined methodology for calculating the heating of aerial power transmission lines. Elektricheskie stantsii [Electric Power Plants]. 2013, no. 9, pp. 54-59 (in Russ.).

7. Guide for Thermal Rating Calculations of Overhead Lines. WG B2.43, Tech. Rep., CIGRE, 2014, 95 p.

8. Mekhanoshin B.I., Shkaptsov V.A., Vasilev Yu.A. Increasing the efficiency of using existing aerial lines based on the analysis of their technical condition and monitoring of wire temperature. Elektro. 2007, no. 6, pp. 37-41 (in Russ.).

9. Gerasimenko A.A., Timofeev G.S., Tikhonovich A.V. The accounting of regime and atmosphere factors in calculation of technical loss of electricity in distributive network. Zhurnal Sibirskogo fed. un-ta. Ser. Tekhnika i tekhnologii [SibFU Jour. Engineering \& Technologies]. 2008, vol. 1, no. 2, pp. 188-206 (in Russ.).

10. Petrova E.V., Bubenchikov A.A., Kirichenko N.V., Ptitsyna E.V. Working out of the losses calculation algorithm in the isolated and not isolated wires of air-lines taking into account operation mode and climatic factors. Omsky nauch. vestn. [Omsk Scientific Bulletin]. Omsk, OmGTU Publ., 2011, no. 3, pp. 201-211 (in Russ.).

11. Balametov A.B., Bayramov M.P. Wire temperature modeling for calculating electric power losses of aerial lines. Problemy energetiki [Energy Sector Problems]. 2013, no. 2, pp. 4-12 (in Russ.).

12. Cecchi V., Leger A.St., Miu K., Nwankpa Ch.O. Incorporating temperature variations into transmission-line models. IEEE Trans. on Power Delivery. 2011, vol. 26, i. 4, pp. 2189-2196.

13. Mo Y., Zhou X., Wang Ya., Liang L. Study on operating status of overhead transmission lines based on wind speed variation. Progress in Electromagnetics Research M. 2017, vol. 60, pp. 111-120.

14. Rahman M., Kiesau M., Cecchi V. Investigating the impacts of conductor temperature on power handling capabilities of transmission lines using a multi-segment line model. SoutheastCon. 2017, pp. 1-7. 